\title{
AMENDMENTS TO THE CONTENT OF AMINO ACIDS IN MEAT OF SLAUGHTER TARINS IN THE PROCESS OF WASHING AND DISINFECTING MEANS
}

\section{Bogatko N. M.}

\section{INTRODUCTION}

State risk-oriented control exercised by veterinary specialists envisages taking measures to check the compliance of market operators with the requirements of the legislation on safety and individual indicators of food quality, as well as eliminating the consequences of non-compliance and holding them accountable for violations of relevant requirements ${ }^{1}$. The risk assessment shall include the identification of a hazardous factor - biological, chemical and physical, the characterization and evaluation of its impact on the safety of the meat of slaughtered animals. Therefore, in order to obtain safe food products, it is necessary to carry out risk-oriented monitoring of the detection of a dangerous chemical factor, namely detergents in slaughtered animals for production and circulation due to breach of terms and timing ${ }^{2}$. Therefore, it is necessary to establish the nutritional value of such meat by its chemical composition, energy value, taste properties and digestibility, and biological value should be taken into account due to the balance of essential amino acids ${ }^{3}$.

Food market operators for their production and circulation must comply with food law at all stages of their production and circulation; to develop, implement and apply permanent procedures based on the principles of the system of analysis of dangerous factors and control at critical points (HACCP systems) ${ }^{4}$. And state control should be carried out on the principle of safety priority in matters of life and human health over any other interests and goals in the sphere of economic activity ${ }^{5}$.

${ }^{1}$ On State Control of Compliance with Food, Feed, Animal By-Products, Animal Health and Welfare : The Law of Ukraine № 2042-VII, 18.05.2017. 43 p.

${ }^{2}$ Bogatko N. Safety and quality of slaughtered animal meat when treated with alkaline detergents. Scientific and Practical Journal of Veterinary Medicine, Livestock Technology and Environmental Management. Kharkiv, 2019. № 4.P. 12-18. DOI: 10.31890/vttp.2019.04.02.

${ }^{3}$ Hulebak K., Schlosser W. Hazard analysis and critical control point (HACCP) history and conceptual overview. Riskanalysis. 2012. № 22 (3). P. 547-552. DOI: $10.1111 / 0272-4332.000383$.

${ }^{4}$ On basis principles and requirements for safety and quality of food : The Law of Ukraine № 1602-VII from 22.07.2014. Approved. Verhovna Rada of Ukraine. 64 p.

${ }_{5}$ The role of hazard- and risk-based approaches in ensuring food safety / S. Barlow et al. Trends in Food Science \& Technology. 2015. Vol. 46. P. 176-188. 
Animal proteins are better balanced in amino acid composition, more responsive to the human body in essential amino acids. The digestibility of animal proteins reaches $70-90 \%$, while the plant protein $-64-75 \%$. Meat proteins provide for the development and metabolism of the body, serve as a material for the construction of cells, tissues and organs, the formation of enzymes and hormones. Researchers say that a person's annual need for high-grade protein is $25 \mathrm{~kg}$, and insufficient protein supply causes impaired brain development, central nervous system, organs of the secretion, circulatory system ${ }^{6}$.

In terms of absolute number of essential amino acids, meat proteins of animals of different species do not differ significantly, although beef is superior to other animal species ${ }^{7}$.

Scientists found that pork and beef pate had higher threonine content (amino acid score of 107 and 112\%, respectively), valine (108 and 112\%), leucine (108 and 111\%), isoleucine (105 and 107\%), as well as lysine (116 and 120\%), which deficiency in children can cause growth retardation, bone calcification disorders, muscle dystrophy ${ }^{8}$. The amino acid score in beef and pork meat pies was within FAO ideal protein units: valine - 5.0; isoleucine -4.0 ; leucine -7.0 ; lysine -5.5 ; threonine -4.0 ; tryptophan 1.0; phenylvinyl + tyrosine -6.0 ; The methionine + cysteine limit was 3.5 (amino acid score in meat pate 85 and $91 \%$ ). Meat products are a source of many selective substances that positively affect the functions of the human body and the addition of certain chemical compounds to the meat product can adversely affect ${ }^{9}$.

Therefore, in order to establish a chemical hazard for the production and circulation of slaughtered animal meat, it is necessary to apply simple chemical studies using rapid tests carried out at the place of the state control measures.

International legislation requires testing of chemicals in foodstuffs to assess the adverse effects and protection of humans, animals and the environment $^{10}$, and to implement an effective monitoring system for

${ }^{6}$ Birta G. Commodity characteristics of pig production. Nutritional value of pork meat. 2019. URL: https://westudents.com.ua >glavy> 90505-8-harchovka-tsnnst-mysasvinins.html.

Nutritional and biological value of meat and meat products. 2019. URL: studopedia.org/13-118914.html.

${ }_{8}$ Borsolyuk L., Wojciechowska S., Shelkova T. Investigation of amino acid composition and relative biological value of functional meat pates. Innovative development of the food industry : Materials VII Intern. Research Practice Conf. November 21, YAN NAR. 2019. P. 19-22.

9 Desarrollo de productos carnicos funcionales : utilizacion deharina de quinua / M. Pena et al. Alimentos, Ciencia e Investigacion. 2015. № 23 (1). P. 21-36.

${ }^{10}$ Council Directive EU 89/320/EEC of 9 June 1988 on the approximation of the laws of the EU Member States relating to the inspection and verification of compliance with established rules for laboratories (UPL) (88/320/EEC) to carry out organizational procedures and conditions under which planned, conducted, recorded and presented the results of tests of chemicals used in industry to assess the effects of such substances on humans, animals and the environment. $16 \mathrm{p}$. 
detecting in the meat of prohibited substances some pollutants from the environment ${ }^{11}$.

Therefore, the question now is to develop express methods of controlling the chemical hazardous factor in the meat of slaughtered animals in the case of detergents and to test the content of amino acids in meat of different quality.

The purpose is to establish the amino acid composition of beef, pork, lamb and goat fresh, questionable freshness and processed detergents.

The task is to evaluate the content of amino acids in the meat of slaughter animals of different quality and in the case of detergent treatment to conceal the signs of poor food.

Material and methods. The study included meat samples of slaughtered animals from the longest back muscle of 126, which were selected from the production and handling capacity of slaughtered animals. which were fresh and questionable and also processed: beef with formalin solution (10\%) and chlorine solution (chlorine activity 3\%); pork - solution of hydrogen peroxide with a mass concentration of $5 \%$ and alkaline detergents; mutton potassium permanganate solution with a mass concentration of 5\%; meat of goat - a solution of acetic acid (10\%), which was selected from the production and circulation facilities in the Kiev region.

It has been previously established by express methods of detection of meat of different quality according to the conventional methods ${ }^{12}$ and by the express patented methods for the establishment of treatment with detergents and disinfectants ${ }^{13}$.

A mixture of concentrated nitric and sulfuric acids in a ratio of 1:25, which was applied to the surface of muscle tissue of pork, beef, lamb, goat, $2,5 \times 3,0 \mathrm{~cm}$ in size and in 4-6 minutes, was used to detect the processing of meat with formalin solution. Set the color change: purple-red (with falsification by formalin solution) or yellow-brown (in the absence of falsification $)^{14}$.

To identify the meat processing of chlorine solution, $2,0-2,1 \mathrm{~cm}^{3}$ of meat-water extract was used in a ratio of $1: 2$, to which reagents were added

${ }^{11}$ Council Directive EU No 23/96 of 29 April 1996 on measures to control individual substances and their residual content in live animals and animal products, adopted in violation of EEC Directives 85/358 and Regulation 89/187/ EEC and 91/664/ EEC. 23 p.

${ }_{12}$ Meat. Methods of chemical and microscopic analysis of freshness. GOST 23392-2016. Date of introduction: 01.01.1980. Change 10.18.2016. Moscow : Standartinform, 2016. 7 p. ; Rules of pre-slaughter veterinary inspection of animals and veterinary-sanitary examination of meat and meat products : Order of the State Department of Veterinary Medicine of 21.06.2002. № 28. $46 \mathrm{p}$.

${ }_{13}$ Bogatko N. Control of the safety of meat of slaughtered animals by falsification by express methods : scientific and methodological recommendations. Bila Tserkva, 2019.26 p.

${ }^{14}$ A method of determining the falsification of meat of slaughtered animals and poultry by formalin treatment / N. Bogatko et al. Patent of Ukraine for utility model 81943, IPC G01N 33/12 (2013.01). № u 201302270 ; claimed 02.25.2013 ; publ. 10.07.2013. Bul. № 13. 4 p. 
sequentially: $0,2-0,3 \mathrm{~cm}^{3}$ of potassium iodide solution with a mass concentration of $5,0 \%, 0,2-0,3 \mathrm{~cm}^{3}$ of a solution of water-soluble starch with a mass concentration of $2,0 \%$ and $2,0-2,1 \mathrm{~cm}^{3}$ of concentrated hydrochloric acid, and after 1-4 minutes the presence of color: blue (by adulteration with a solution) chlorine) or colorless (in the absence of falsification) ${ }^{15}$.

$0,5-0,6 \mathrm{~cm}^{3}$ of concentrated sulfuric acid and $0,2-0,4 \mathrm{~cm}^{3}$ of iodidepotassium starch applied to the surface of muscle tissue of pork, beef, lamb, goat were used to detect the treatment of meat with hydrogen peroxide solution. 1,5 x 2,0 cm in size and after 1-5 minutes the presence of color was determined: light blue (by adulteration with a solution of hydrogen peroxide) or without color formation (in the absence of adulteration) ${ }^{16}$.

$0,5-0,6 \mathrm{~cm}^{3}$ of sodium hydroxide solution with a mass concentration of $0,1 \mathrm{~mol} / \mathrm{dm}^{3}$ and $0,1-0,2 \mathrm{~cm}^{3}$ indicator of an alcohol solution of phenolphthalein with a mass concentration of $1 \%$ were used to detect the processing of meat with a solution of acetic acid. was applied to the surface of the muscle tissue of pork, beef, lamb, goat with an area of 2,0 x 2,5 cm and after $0,5-1,0$ minutes established the presence or absence of color: pink (by adulteration with acetic acid solution) or without color formation (in the absence of falsification) ${ }^{17}$.

To identify the processing of meat with a solution of potassium permanganate used $0,4-0,5 \mathrm{~cm}^{3}$ solution of sulfuric acid with a mass concentration of $0,5 \mathrm{~mol} / \mathrm{dm}^{3}$, which was applied to the surface of the muscle tissue of pork, beef, lamb, goat size $2,0 \times 2,5 \mathrm{~cm}$ and after $0,5-1,0$ minutes the presence of color was determined: slightly pink (with falsification by a solution of potassium permanganate) or without color formation (in the absence of falsification) ${ }^{18}$.

To identify the processing of meat with alkaline detergent solutions used $0,2-0,3 \mathrm{~cm}^{3}$ alcohol solution of bromothymol blue with a mass concentration of $0.04 \%$, which was applied to the surface of the muscle tissue of pork, beef, lamb, goat size $2,0 \times 2,5 \mathrm{~cm}$ and after $2-3$ seconds established the presence of a light yellow color (negative reaction - no falsification) or the

15 The method of determining the adulteration of meat of slaughtered animals and poultry by chlorine treatment / N. Bogatko et al. Patent of Ukraine for utility model 81944, IPC G01N 33/12 (2013.01). № u 2013 02271; claimed 02.25.2013 ; publ. 10.07.2013. Bul. № 13. 4 p.

${ }^{16}$ The method of determining the adulteration of meat of slaughtered animals and poultry by treatment with hydrogen peroxide / N. Bogatko et al. Patent of Ukraine for utility model 81945, IPC G01N 33/12 (2013.01). № u 201302273 ; claimed 02.25.2013 ; publ 10.07 .2013 . Bul. № 13.3 p.

${ }_{17}$ The method of determining the adulteration of meat of slaughtered animals and poultry by treatment with acetic acid / M. Bogatko et al. Patent of Ukraine for utility model 102019, IPC G01N 33/12 (2006.01). № u 201503749 ; claimed 21.04.2015; publ. 12.10.2015. Bul. № 19. 4 p.

${ }_{18}$ The method of determining the adulteration of the meat of slaughtered animals and poultry by treatment with a solution of potassium permanganate / M. Bogatko et al. Patent of Ukraine for utility model 102020, IPC G01N 33/12 (2006.01). № u 201503750 ; claimed 21.04.2015; publ. 12.10.2015. Bul. № 19. 3 p. 
presence of blue-blue color of different intensity depending on the amount of alkaline detergents added: light blue (positive reaction) - the presence of alkaline detergents on the surface of muscle tissue up to 5,0\%; blue-blue (positive reaction) - presence of application of alkaline detergents on the surface of muscle tissue more than $5,1 \%{ }^{19}$.

Tests were carried out to determine the amino acid composition of meat of slaughtered animals of different quality (fresh, doubtful freshness and doubtful freshness for treatment with different detergents) in the Department of Lipid Biochemistry, Chromatography Unit of the Biochemistry Institute of the name of O.V. Palladin NAS of Ukraine by ion-exchange liquid chromatography on a TTT 339 automatic analyzer (production Czech Republic, Prague) by hydrolysis of the meat sample with hydrochloric acid and its deproteinization (protein deposition) to obtain the free amino acid extract and further separating the amino acids on the ion-exchange columns using lithium citrate buffers as eluent and calculating the peak area of each amino acid on the chromatogram and comparing it with the peak area of the amino acids at a known concentration. Comparison of these areas made the calculation of the absolute amount of amino acids in the analyzed sample. State-owned enterprise "Ukrmetrteststandart" recognizes the measuring capabilities of the Testing Biological Center of the Biochemistry Institute of the name of O.V. Palladinof the National Academy of Sciences of Ukraine under the certificate № PT-221/17 of 12.10.2017.

\section{Establishment of amino acid composition in beef and pork for their treatment with detergents}

Ion exchange column chromatography is used in an important area to determine the qualitative and quantitative analysis of peptides and proteins, which gives a valuable characterization of molecules, as well as to establish the amino acid composition of meat of slaughtered animals, which allows to track changes in meat that occur under the impact of detergents in the case of intentional application to conceal the poor quality of these foods. The results of amino acid content of beef of different quality: fresh, questionable freshness and treated with formalin and chlorine solutions are presented in table 1 .

${ }^{19}$ The method of determining the adulteration of the meat of slaughtered animals and poultry by treatment with alkaline detergents / M. Bogatko et al. Patent of Ukraine for utility model 116831, IPC G01N 33/12 (2006.01). № u 201612243 ; claimed 02.12.2016 ; publ. 12.06.2017. Bul. № 11. 4 p. 
Table 1

Amino acid composition of beef of different quality, $M \pm m, n=36$

\begin{tabular}{|c|c|c|c|c|c|c|c|c|}
\hline \multirow{3}{*}{$\begin{array}{l}\text { Amino } \\
\text { acids }\end{array}$} & \multicolumn{8}{|c|}{ Beef of different quality } \\
\hline & \multicolumn{2}{|c|}{$\begin{array}{c}\text { Fresh beef } \\
\text { (control) }\end{array}$} & \multicolumn{2}{|c|}{$\begin{array}{c}\text { Beef of dubious } \\
\text { freshness }\end{array}$} & \multicolumn{2}{|c|}{$\begin{array}{c}\text { Beef treated with } \\
\text { formalin solution } \\
(10 \%)\end{array}$} & \multicolumn{2}{|c|}{$\begin{array}{c}\text { Beef treated with } \\
\text { chlorine solution } \\
\text { (chlorine activity } 3 \% \text { ) }\end{array}$} \\
\hline & $\begin{array}{c}\text { quantit } \\
\text { y, mg }\end{array}$ & $\begin{array}{c}\text { \% by } \\
\text { mg }\end{array}$ & $\begin{array}{l}\text { quantity, } \\
\text { mg }(* * *)\end{array}$ & $\begin{array}{c}\text { \% by } \\
\text { mg }\end{array}$ & $\begin{array}{l}\text { quantity, } \\
\text { mg (***) }\end{array}$ & $\begin{array}{c}\text { \% by } \\
\text { mg }\end{array}$ & $\begin{array}{l}\text { quantity, } \\
\text { mg (***) }\end{array}$ & $\begin{array}{c}\text { \% by } \\
\text { mg }\end{array}$ \\
\hline Lysine & $\begin{array}{c}1,810 \pm \\
0,0020\end{array}$ & 9,36 & $\begin{array}{l}1,627 \pm \\
0,0040\end{array}$ & 8,59 & $\begin{array}{l}1,672 \pm \\
0,0040\end{array}$ & 10,34 & $\begin{array}{l}1,518 \pm \\
0,0030\end{array}$ & 8,34 \\
\hline Histidine & $\begin{array}{c}0,674 \pm \\
0,0020\end{array}$ & 3,49 & $\begin{array}{c}0,727 \pm \\
0,0060\end{array}$ & 3,84 & $\begin{array}{c}0,369 \pm \\
0,0018\end{array}$ & 2,28 & $\begin{array}{c}0,711 \pm \\
0,0021\end{array}$ & 3,90 \\
\hline Arginine & $\begin{array}{l}1,114 \pm \\
0,0040\end{array}$ & 5,76 & $\begin{array}{l}1,021 \pm \\
0,0100\end{array}$ & 5,39 & $\begin{array}{c}0,865 \pm \\
0,0030\end{array}$ & 5,34 & $\begin{array}{l}1,018 \pm \\
0,0025\end{array}$ & 5,59 \\
\hline O-Proline & $\begin{array}{c}0,140 \pm \\
0,0040\end{array}$ & 0,72 & $\begin{array}{c}0,155 \pm \\
0,0020\end{array}$ & 0,82 & $\begin{array}{c}0,126 \pm \\
0,0019\end{array}$ & 0,78 & $\begin{array}{c}0,146 \pm \\
0,0024\end{array}$ & 0,80 \\
\hline $\begin{array}{l}\text { Aspartic } \\
\text { acid }\end{array}$ & $\begin{array}{l}1,310 \pm \\
0,0010\end{array}$ & 6,77 & $\begin{array}{l}1,197 \pm \\
0,0020\end{array}$ & 6,32 & $\begin{array}{l}1,266 \pm \\
0,0037\end{array}$ & 7,83 & $\begin{array}{l}1,128 \pm \\
0,0027\end{array}$ & 6,19 \\
\hline Threonine & $\begin{array}{c}0,921 \pm \\
0,0030\end{array}$ & 4,76 & $\begin{array}{c}0,862 \pm \\
0,0020\end{array}$ & 4,55 & $\begin{array}{c}0,783 \pm \\
0,0031\end{array}$ & 4,840 & $\begin{array}{c}0,922 \pm \\
0,0078\end{array}$ & 5,06 \\
\hline Serine & $\begin{array}{c}0,794 \pm \\
0,0020\end{array}$ & 4,11 & $\begin{array}{c}0,757 \pm \\
0,0020\end{array}$ & 3,99 & $\begin{array}{c}0,674 \pm \\
0,0030\end{array}$ & 4,16 & $\begin{array}{c}0,804 \pm \\
0,0027\end{array}$ & 4,41 \\
\hline $\begin{array}{l}\text { Glutamic } \\
\text { acid }\end{array}$ & $\begin{array}{c}3,597 \pm \\
0,0030\end{array}$ & 18,61 & $\begin{array}{c}3,443 \pm \\
0,0030\end{array}$ & 18,18 & $\begin{array}{c}2,872 \pm \\
0,0060\end{array}$ & 17,75 & $\begin{array}{c}2,816 \pm \\
0,0051\end{array}$ & 15,46 \\
\hline Proline & $\begin{array}{c}0,828 \pm \\
0,0060\end{array}$ & 4,28 & $\begin{array}{c}0,893 \pm \\
0,0020 \\
\end{array}$ & 4,71 & $\begin{array}{c}0,604 \pm \\
0,0020\end{array}$ & 3,73 & $\begin{array}{c}0,797 \pm \\
0,0028 \\
\end{array}$ & 4,38 \\
\hline Glycine & $\begin{array}{c}1,038 \pm \\
0,0050\end{array}$ & 5,37 & $\begin{array}{l}1,035 \pm \\
0,0020\end{array}$ & 5,46 & $\begin{array}{l}1,080 \pm \\
0,0022\end{array}$ & 6,68 & $\begin{array}{c}0,936 \pm \\
0,0030 \\
\end{array}$ & 5,14 \\
\hline Alanine & $\begin{array}{l}1,229 \pm \\
0,0070\end{array}$ & 6,36 & $\begin{array}{c}1,148 \pm \\
0,0030\end{array}$ & 6,06 & $\begin{array}{l}1,149 \pm \\
0,0023\end{array}$ & 7,10 & $\begin{array}{l}1,138 \pm \\
0,0020\end{array}$ & 6,25 \\
\hline Cystine & $\begin{array}{c}0,175 \pm \\
0,0040 \\
\end{array}$ & 0,90 & $\begin{array}{c}0,119 \pm \\
0,0010 \\
\end{array}$ & 0,63 & $\begin{array}{c}0,090 \pm \\
0,0016\end{array}$ & 0,56 & $\begin{array}{l}0,169 \pm \\
0,0025\end{array}$ & 0,93 \\
\hline Valine & $\begin{array}{c}0,798 \pm \\
0,0010\end{array}$ & 4,13 & $\begin{array}{c}0,997 \pm \\
0,0030\end{array}$ & 5,26 & $\begin{array}{c}0,940 \pm \\
0,0023\end{array}$ & 5,81 & $\begin{array}{l}1,043 \pm \\
0,0032\end{array}$ & 5,73 \\
\hline Methionine & $\begin{array}{l}0,558 \pm \\
0,0050\end{array}$ & 2,89 & $\begin{array}{c}0,548 \pm \\
0,0040\end{array}$ & 2,89 & $\begin{array}{c}0,433 \pm \\
0,0016\end{array}$ & 2,68 & $\begin{array}{c}0,597 \pm \\
0,0031\end{array}$ & 3,28 \\
\hline Isoleucine & $\begin{array}{c}0,852 \pm \\
0,0080\end{array}$ & 4,41 & $\begin{array}{c}0,940 \pm \\
0,0040\end{array}$ & 4,96 & $\begin{array}{c}0,847 \pm \\
0,0027\end{array}$ & 5,24 & $\begin{array}{c}0,958 \pm \\
0,0026\end{array}$ & 5,26 \\
\hline Leucine & $\begin{array}{l}1,905 \pm \\
0,0090\end{array}$ & 9,86 & $\begin{array}{l}1,823 \pm \\
0,0040\end{array}$ & 9,62 & $\begin{array}{l}1,551 \pm \\
0,0040\end{array}$ & 9,59 & $\begin{array}{l}1,793 \pm \\
0,0040\end{array}$ & 9,84 \\
\hline Tyrosine & $\begin{array}{c}0,760 \pm \\
0,0050\end{array}$ & 3,93 & $\begin{array}{c}0,755 \pm \\
0,0020\end{array}$ & 3,99 & $\begin{array}{c}0,099 \pm \\
0,0030\end{array}$ & 0,61 & $\begin{array}{c}0,777 \pm \\
0,0028\end{array}$ & 4,27 \\
\hline $\begin{array}{c}\text { Phenylala } \\
\text { nine }\end{array}$ & $\begin{array}{c}0,830 \pm \\
0,0090\end{array}$ & 4,30 & $\begin{array}{c}0,896 \pm \\
0,0060\end{array}$ & 4,73 & $\begin{array}{c}0,757 \pm \\
0,0021\end{array}$ & 4,68 & $\begin{array}{c}0,945 \pm \\
0,0032\end{array}$ & 5,19 \\
\hline $\begin{array}{c}\text { Summary } \\
\text { content }\end{array}$ & $\begin{array}{c}19,332 \\
\pm 0,076\end{array}$ & 100,0 & $\begin{array}{c}18,942 \pm \\
0,056 * * *\end{array}$ & 100,0 & $\begin{array}{c}16,178 \pm \\
0,044 * * *\end{array}$ & 100,0 & $\begin{array}{c}18,216 \pm \\
0,044 * * *\end{array}$ & 100,0 \\
\hline
\end{tabular}

Note: $* * *-p \leq 0,001$ - for all amino acids by the quantitative content in $m g$ of beef of questionable freshness and treated with formalin and chlorine solutions.

The total amino acid content of beef of questionable freshness was $18,942 \pm 0,056 \mathrm{mg}$, which was 1,02 times $(\mathrm{p} \leq 0,001)$ significantly decreased compared to the amino acid composition of fresh beef. This is characterized by a likely decrease in the quantitative content of such amino acids as: 
cystine $-1,47$ times $(p \leq 0,001)$, lysine $-1,11$ times $(p \leq 0,001)$, arginine and aspartic acid $-1,09$ times $(\mathrm{p}<0,001)$, threonine and alanine $-1,07$ times $(\mathrm{p} \leq 0,001)$, serine $-1,05$ times $(\mathrm{p} \leq 0,001)$, glutamic acid and leucine 1,04 times $(p<0,001)$, methionine $-1,02$ times $(p \leq 0,001)$.

But at the same time, the quantitative content of valine increased by 1,25 times $(p \leq 0,001)$, O-proline - by 1,11 times $(p \leq 0,001)$, isoleucine by 1,10 times $(p \leq 0,001)$ and histidine, proline, phenylalanine $-1,08$ times ( $p \leq 0,001)$ compared to fresh beef, and the difference in indicators was significant. There is no significant difference in glycine and tyrosine content.

In the case of formalin solution (10\%) beef of dubious freshness, the total amino acid content was significantly reduced by 1,19 times $(\mathrm{p} \leq 0,001)$ compared to the control (fresh beef) indicators due to a likely decrease in the number of 15 amino acids: tyrosine - by 7,68 ( $\mathrm{p} \leq 0,001)$, cystine 1,94 times $(\mathrm{p} \leq 0,001)$, histidine $-1,83$ times $(\mathrm{p} \leq 0,001)$, proline $-1,37$ times $(\mathrm{p} \leq 0,001)$, arginine and methionine - by 1,29 times $(\mathrm{p} \leq 0,001)$, glutamic acid $-1,25$ times $(\mathrm{p} \leq 0,001)$, leucine $-1,23$ times $(\mathrm{p} \leq 0,001)$, threonine and serine $-1,18$ times $(\mathrm{p} \leq 0,001)$, phenylalanine $-1,10$ times $(\mathrm{p} \leq 0,001)$, O-proline $-1,11$ times $(\mathrm{p} \leq 0,001)$, lysine $-1,08$ times $(\mathrm{p} \leq 0,001)$, alanine $-1,07$ times $(\mathrm{p} \leq 0,001$ aspartic acid $-1,03$ times $(\mathrm{p} \leq 0,001)$. No significant difference in isoleucine content was established. However, there was a probable increase in valine by 1,18 times $(p \leq 0,001)$ and glycine by 1,04 times $(\mathrm{p} \leq 0,001)$ compared to control values.

When treating beef of dubious freshness with a chlorine solution (chlorine activity of 3\%), the total amino acid content was significantly reduced by 1,06 times $(\mathrm{p} \leq 0,001)$ compared to the controls (fresh beef). Namely, there was a probable decrease in the quantitative content of such amino acids as: glutamic acid - by 1,28 times $(\mathrm{p} \leq 0,001)$, lysine - by 1,19 times $(\mathrm{p} \leq 0,001)$, aspartic acid - by 1,16 times $(\mathrm{p} \leq 0,001)$, glycine 1,11 times $(\mathrm{p} \leq 0,001)$, arginine $-1,09$ times $(\mathrm{p} \leq 0,001)$, alanine 1,08 times $(p \leq 0,001)$, leucine $-1,06$ times $(p \leq 0,001)$, as well as proline and cystine $-1,04$ times $(\mathrm{p} \leq 0,001)$ compared to control values.

Significant increase in beef treated with chlorine solution (chlorine activity of $3 \%$ ) of some quantitative amino acid content, such as: valine 1,31 times $(\mathrm{p} \leq 0,001)$, phenylalanine $-1,14$ times $(\mathrm{p} \leq 0,001)$, isoleucine in 1,12 times $(p \leq 0,001)$, methionine $-1,07$ times $(p \leq 0,001)$, histidine 1,05 times $(\mathrm{p} \leq 0,001)$, O-proline $-1,04$ times $(\mathrm{p} \leq 0,001)$, tyrosine 1,02 times $(\mathrm{p} \leq 0,001)$, and a slight significant increase in serine and threonine, respectively $-1,012$ times $(p \leq 0,001)$ and 1,001 times $(p \leq 0,001)$ compared to control values.

In table 2 summarizes the content scor of beef cuts of different quality. 
The content scor in amino acids $(\%), M \pm m, n=36$

\begin{tabular}{|c|c|c|c|c|}
\hline $\begin{array}{l}\text { Amino } \\
\text { acids }\end{array}$ & $\begin{array}{l}\text { Fresh beef } \\
\text { (control) }\end{array}$ & $\begin{array}{c}\text { Beef of } \\
\text { dubious } \\
\text { freshness }\end{array}$ & $\begin{array}{c}\text { Beef treated } \\
\text { with formalin } \\
\text { solution }(\mathbf{1 0 \%}) \\
\end{array}$ & $\begin{array}{c}\begin{array}{c}\text { Beef treated with } \\
\text { chlorine solution } \\
\text { (chlorine activity } 3 \% \text { ) }\end{array} \\
\end{array}$ \\
\hline Lysine & $170 \pm 0,52$ & $\begin{array}{l}156,00 \pm \\
0,57 * * *\end{array}$ & $\begin{array}{l}188,00 \pm \\
0,62 * * *\end{array}$ & $152,00 \pm 0,42 * * *$ \\
\hline Threonine & $\begin{array}{c}119,00 \pm \\
0,47\end{array}$ & $\begin{array}{c}114,00 \pm 0,59 \\
* * *\end{array}$ & $121,00 \pm 0,71^{*}$ & $127,00 \pm 0,39 * * *$ \\
\hline Cystine & $\begin{array}{c}108,00 \pm \\
0,54\end{array}$ & $\begin{array}{l}101,00 \pm \\
0,54 * * *\end{array}$ & $92,00 \pm 0,83 * * *$ & $120,00 \pm 0,82 * * *$ \\
\hline Valin & $\begin{array}{c}83,00 \pm \\
0,42\end{array}$ & $\begin{array}{l}105,00 \pm \\
0,82 * * *\end{array}$ & $\begin{array}{l}116,00 \pm \\
0,61 * * *\end{array}$ & $115,00 \pm 0,41 * * *$ \\
\hline Isoleucine & $\begin{array}{c}110,00 \pm \\
0,66\end{array}$ & $\begin{array}{l}124,00 \pm \\
0,90 * * *\end{array}$ & $\begin{array}{l}131,00 \pm \\
0,70^{* * * *}\end{array}$ & $131,00 \pm 0,44 * * *$ \\
\hline Leucine & $\begin{array}{c}141,00 \pm \\
0,67\end{array}$ & $\begin{array}{l}137,00 \pm \\
0,58 * * *\end{array}$ & $\begin{array}{l}137,00 \pm \\
0,67 * * *\end{array}$ & $141,00 \pm 0,51 * * *$ \\
\hline Tyrosine & $\begin{array}{c}137,00 \pm \\
0,51\end{array}$ & $\begin{array}{l}145,00 \pm \\
0,59 * * *\end{array}$ & $88,00 \pm 0,49 * * *$ & $158,00 \pm 0,47 * * *$ \\
\hline
\end{tabular}

Note: $*-p \leq 0,05 ; * * *-p \leq 0,001$.

In beef of questionable freshness and questionable freshness of chlorine solution treated (chlorine activity of 3\%), the lysine scor was significantly decreased respectively by 1,09 times $(\mathrm{p} \leq 0,001)$ and 1,12 times $(\mathrm{p} \leq 0,001)$, and in formalin-treated beef $(10 \%)-1,11$ times $(\mathrm{p}<0,001)$ compared to fresh beef. The threonine scor of beef of questionable freshness was probably decreased by 1,04 times $(\mathrm{p} \leq 0,001)$, and in beef treated with chlorine and formalin solutions it was increased, respectively, by 1,07 times $(p \leq 0,001)$ and 1,02 times $(p \leq 0,05)$ compared to the indicators of fresh beef.

In beef of questionable freshness and formalin-treated beef, the cystinescor was significantly decreased - by 1,07 times $(p \leq 0,001)$ and 1,17 times $(p \leq 0,001)$, and in the treatment of beef with chlorine solution, it was increased by 1,11 times $(p \leq 0,001)$. Also, the valine scor in beef of questionable freshness and questionable freshness of formalin and chlorine treated with the solution was significantly increased, respectively - by 1,27 times $(\mathrm{p} \leq 0,001), 1,40$ times $(\mathrm{p} \leq 0,001)$ and 1,39 times $(\mathrm{p} \leq 0,001)$; the isoleucine scor was also likely increased by 1.13 -fold $(\mathrm{p} \leq 0,001), 1,19$-fold $(\mathrm{p} \leq 0,001)$ and 1,19 -fold $(\mathrm{p} \leq 0,001)$, respectively. The leucine scor in beef of questionable freshness and treated with formalin solution was probably reduced by 1,03 -fold $(\mathrm{p} \leq 0,001)$, and in beef treated with chlorine solution was constant and did not change $-141 \pm 0,51 \%$. The tyrosine scor in beef of questionable freshness and treated with chlorine solution increased significantly, respectively, by 1,06 times $(\mathrm{p} \leq 0,001)$ and 1,15 times $(p \leq 0,001)$, and in formalin-treated beef significantly decreased by 1,03 times $(p \leq 0,001)$ compared to fresh beef.

Analysis of the amino acid composition of pork of different quality is presented in table 3 . 
Table 3

Amino acid composition of pork of different quality, $M \pm m, n=36$

\begin{tabular}{|c|c|c|c|c|c|c|c|c|}
\hline \multirow{3}{*}{$\begin{array}{l}\text { Amino } \\
\text { acids }\end{array}$} & \multicolumn{8}{|c|}{ Pork of different quality } \\
\hline & \multicolumn{2}{|c|}{$\begin{array}{l}\text { Fresh pork } \\
\text { (control) }\end{array}$} & \multicolumn{2}{|c|}{$\begin{array}{l}\text { Pork of dubious } \\
\text { freshness }\end{array}$} & \multicolumn{2}{|c|}{$\begin{array}{c}\text { Pork treated with } \\
\text { hydrogen peroxide } \\
(5 \%)\end{array}$} & \multicolumn{2}{|c|}{$\begin{array}{c}\text { The pork is treated } \\
\text { with alkaline detergents } \\
\text { and disinfectants }\end{array}$} \\
\hline & $\begin{array}{l}\text { quantity, } \\
\text { mg }\end{array}$ & $\begin{array}{c}\% \\
\text { by } \\
\text { mg }\end{array}$ & $\begin{array}{l}\text { quantity, } \\
\text { mg (***) }\end{array}$ & $\begin{array}{c}\% \text { by } \\
\text { mg }\end{array}$ & $\begin{array}{l}\text { quantity, } \\
\text { mg (***) }\end{array}$ & $\begin{array}{c}\% \text { by } \\
\text { mg }\end{array}$ & $\begin{array}{c}\text { quantity, mg } \\
(* * *)\end{array}$ & $\begin{array}{c}\text { \% by } \\
\text { mg }\end{array}$ \\
\hline Lysine & $\begin{array}{l}1,825 \pm \\
0,0040\end{array}$ & $\begin{array}{c}9,1 \\
4\end{array}$ & $\begin{array}{l}1,526 \pm \\
0,0031\end{array}$ & 8,01 & $\begin{array}{l}1,950 \pm \\
0,0054\end{array}$ & 9,87 & $\begin{array}{l}1,770 \pm \\
0,0023\end{array}$ & 8,99 \\
\hline Histidine & $\begin{array}{l}0,915 \pm \\
0,0023\end{array}$ & $\begin{array}{c}4,5 \\
8 \\
\end{array}$ & $\begin{array}{l}1,117 \pm \\
0,0020\end{array}$ & 5,86 & $\begin{array}{c}0,862 \pm \\
0,0037\end{array}$ & 4,36 & $\begin{array}{l}0,843 \pm \\
0,0045\end{array}$ & 4,28 \\
\hline Arginine & $\begin{array}{c}0,148 \pm \\
0,0021\end{array}$ & $\begin{array}{c}5,7 \\
5\end{array}$ & $\begin{array}{l}1,069 \pm \\
0,0020\end{array}$ & 5,61 & $\begin{array}{c}0,944 \pm \\
0,0034\end{array}$ & 4,77 & $\begin{array}{l}0,945 \pm \\
0,0034\end{array}$ & 4,80 \\
\hline O-Proline & $\begin{array}{l}0,066 \pm \\
0,0021\end{array}$ & $\begin{array}{c}0,3 \\
3\end{array}$ & $\begin{array}{l}0,117 \pm \\
0,0050\end{array}$ & 0,61 & $\begin{array}{c}0,157 \pm \\
0,0030\end{array}$ & 0,79 & $\begin{array}{c}0,113 \pm \\
0,0020\end{array}$ & 0,58 \\
\hline $\begin{array}{l}\text { Aspartic } \\
\text { acid }\end{array}$ & $\begin{array}{l}1,381 \pm \\
0,0040\end{array}$ & $\begin{array}{c}6,9 \\
2 \\
\end{array}$ & $\begin{array}{l}1,060 \pm \\
0,0020\end{array}$ & 5,57 & $\begin{array}{l}1,635 \pm \\
0,0052\end{array}$ & 8,27 & $\begin{array}{l}1,343 \pm \\
0,0040\end{array}$ & 6,82 \\
\hline Threonine & $\begin{array}{c}0,913 \pm \\
0,0071\end{array}$ & $\begin{array}{c}4,5 \\
7\end{array}$ & $\begin{array}{c}0,878 \pm \\
0,0060\end{array}$ & 4,61 & $\begin{array}{l}1,009 \pm \\
0,0045\end{array}$ & 5,10 & $\begin{array}{l}1,075 \pm \\
0,0034\end{array}$ & 5,46 \\
\hline Serine & $\begin{array}{c}0,795 \pm \\
0,0090\end{array}$ & $\begin{array}{c}3,9 \\
8\end{array}$ & $\begin{array}{c}0,763 \pm \\
0,0010\end{array}$ & 4,01 & $\begin{array}{c}0,833 \pm \\
0,0033\end{array}$ & 4,21 & $\begin{array}{l}0,907 \pm \\
0,0017\end{array}$ & 4,61 \\
\hline $\begin{array}{c}\text { Glutamic } \\
\text { acid }\end{array}$ & $\begin{array}{c}3,891 \pm \\
0,0032 \\
\end{array}$ & $\begin{array}{l}19 \\
48 \\
\end{array}$ & $\begin{array}{c}3,095 \pm \\
0,0020\end{array}$ & 16,26 & $\begin{array}{c}3,526 \pm \\
0,0060 \\
\end{array}$ & 17,83 & $\begin{array}{c}3,399 \pm \\
0,0043\end{array}$ & 17,27 \\
\hline Proline & $\begin{array}{c}0,604 \pm \\
0,0021\end{array}$ & $\begin{array}{c}3,0 \\
2\end{array}$ & $\begin{array}{c}0,958 \pm \\
0,0030\end{array}$ & 5,03 & $\begin{array}{c}0,664 \pm \\
0,0049\end{array}$ & 3,36 & $\begin{array}{c}0,755 \pm \\
0,0028\end{array}$ & 3,84 \\
\hline Glycine & $\begin{array}{l}1,095 \pm \\
0,0020\end{array}$ & $\begin{array}{c}5,4 \\
8\end{array}$ & $\begin{array}{c}0,925 \pm \\
0,0020\end{array}$ & 4,86 & $\begin{array}{c}0,903 \pm \\
0,0038\end{array}$ & 4,57 & $\begin{array}{c}0,970 \pm \\
0,0033\end{array}$ & 4,93 \\
\hline Alanine & $\begin{array}{l}1,256 \pm \\
0,0034\end{array}$ & $\begin{array}{c}6,2 \\
9\end{array}$ & $\begin{array}{l}0,985 \pm \\
0,0020\end{array}$ & 5,17 & $\begin{array}{l}1,128 \pm \\
0,0023\end{array}$ & 5,70 & $\begin{array}{l}0,866 \pm \\
0,0025\end{array}$ & 4,40 \\
\hline Cystine & $\begin{array}{c}0,123 \pm \\
0,0032\end{array}$ & $\begin{array}{c}0,6 \\
1\end{array}$ & $\begin{array}{c}0,113 \pm \\
0,0020\end{array}$ & 0,59 & $\begin{array}{c}0,137 \pm \\
0,0027\end{array}$ & 0,69 & $\begin{array}{c}0,152 \pm \\
0,0030\end{array}$ & 0,77 \\
\hline Valine & $\begin{array}{l}0,863 \pm \\
0,0063\end{array}$ & $\begin{array}{c}4,3 \\
2\end{array}$ & $\begin{array}{l}1,011 \pm \\
0,0020\end{array}$ & 5,31 & $\begin{array}{l}1,018 \pm \\
0,0026\end{array}$ & 5,15 & $\begin{array}{l}1,124 \pm \\
0,0028\end{array}$ & 5,71 \\
\hline Methionine & $\begin{array}{c}0,574 \pm \\
0,0031\end{array}$ & $\begin{array}{c}2,8 \\
7\end{array}$ & $\begin{array}{c}0,776 \pm \\
0,0030\end{array}$ & 4,07 & $\begin{array}{c}0,652 \pm \\
0,0042\end{array}$ & 3,30 & $\begin{array}{c}0,752 \pm \\
0,0049\end{array}$ & 3,82 \\
\hline Isoleucine & $\begin{array}{c}0,938 \pm \\
0,0024\end{array}$ & $\begin{array}{c}4,7 \\
0\end{array}$ & $\begin{array}{l}1,044 \pm \\
0,0040\end{array}$ & 5,48 & $\begin{array}{c}0,980 \pm \\
0,0024\end{array}$ & 4,96 & $\begin{array}{l}1,042 \pm \\
0,0050\end{array}$ & 5,29 \\
\hline Leucine & $\begin{array}{l}1,977 \pm \\
0,0022\end{array}$ & $\begin{array}{c}9,9 \\
0\end{array}$ & $\begin{array}{l}1,689 \pm \\
0,0010\end{array}$ & 8,87 & $\begin{array}{l}1,737 \pm \\
0,0044\end{array}$ & 8,79 & $\begin{array}{l}1,880 \pm \\
0,0026\end{array}$ & 9,55 \\
\hline Tyrosine & $\begin{array}{c}0,766 \pm \\
0,0023\end{array}$ & $\begin{array}{c}3,8 \\
3 \\
\end{array}$ & $\begin{array}{c}0,905 \pm \\
0,0020\end{array}$ & 4,75 & $\begin{array}{c}0,760 \pm \\
0,0021\end{array}$ & 3,84 & $\begin{array}{c}0,814 \pm \\
0,0040\end{array}$ & 4,14 \\
\hline $\begin{array}{c}\text { Phenylalani } \\
\text { ne }\end{array}$ & $\begin{array}{l}0,841 \pm \\
0,0042\end{array}$ & $\begin{array}{c}4,2 \\
1\end{array}$ & $\begin{array}{l}1,015 \pm \\
0,0020\end{array}$ & 5,33 & $\begin{array}{c}0,880 \pm \\
0,0028\end{array}$ & 4,45 & $\begin{array}{l}0,933 \pm \\
0,0064\end{array}$ & 4,74 \\
\hline $\begin{array}{c}\text { Summary } \\
\text { content }\end{array}$ & $\begin{array}{c}19,970 \pm \\
0,051\end{array}$ & $\begin{array}{l}10 \\
0,0\end{array}$ & $\begin{array}{c}19,047 \pm \\
0,044 * * *\end{array}$ & 100,0 & $\begin{array}{c}19,775 \pm \\
0,040 * * *\end{array}$ & 100,0 & $\begin{array}{c}19,683 \pm \\
0,030 * * *\end{array}$ & 100,0 \\
\hline
\end{tabular}

Note: $* * *-p \leq 0,001-$ for all amino acids by the quantitative content in mg of pork of dubious freshness and treated with hydrogen peroxide solution and alkaline detergent and disinfectants.

The total amino acid content of fresh pork was $19,970 \pm 0,051$, which was $100 \% \mathrm{mg}$. And in pork of doubtful freshness, it probably decreased by 1,05 times $(\mathrm{p} \leq 0,001)$.

A significant reduction of amino acids in pork of doubtful degree was found compared to indicators of fresh pork such as: aspartic acid - 
1,30 times $(\mathrm{p} \leq 0,001)$, alanine $-1,28$ times $(\mathrm{p} \leq 0,001)$, glutamic acid 1,23 times $(\mathrm{p}<0,001)$, lysine $-1,20$ times $(\mathrm{p}<0,001)$, glycine $-1,18$ times $(\mathrm{p} \leq 0,001)$, leucine $-1,17$ times $(\mathrm{p} \leq 0,001)$, cystine $-1,09$ times $(\mathrm{p} \leq 0,001)$, threonine and serine $-1,04$ times $(\mathrm{p} \leq 0,001)$.

In addition, it was found that by quantitative content some amino acids probably increased in pork of dubious freshness, namely: arginine 7,22 times $(\mathrm{p} \leq 0,001)$, O-proline $-1,77$ times $(\mathrm{p} \leq 0,001)$, proline 1,59 times $(\mathrm{p} \leq 0,001)$, methionine $-1,35$ times $(\mathrm{p} \leq 0,001)$, histidine 1,22 times $(\mathrm{p} \leq 0,001)$, phenylalanine $-1,20$ times $(\mathrm{p} \leq 0,001)$, tyrosine 1,18 times $(p \leq 0,001)$, valine $-1,17$ times $(p \leq 0,001)$, isoleucine 1,11 times $(\mathrm{p} \leq 0,001)$ compared to control indicators.

When treating pork of questionable freshness with a solution of hydrogen peroxide with a mass concentration of $5 \%$, the total amino acid content was $19,775 \pm 0,040$, which is 1,01 times $(p \leq 0,001)$ less than the indicators of fresh pork and 1,04 times more than the indicators of doubtful the degree of freshness of pork. The amino acid composition of meat treated with hydrogen peroxide was characterized by a significant decrease in glycine by 1,21 times $(\mathrm{p} \leq 0,001)$, leucine - by 1,14 times $(\mathrm{p} \leq 0,001)$, alanine - by 1,11 times $(\mathrm{p} \leq 0,001)$, glutamic acid $-1,10$ times $(\mathrm{p} \leq 0,001)$, histidine 1,06 times $(\mathrm{p} \leq 0,001)$, and tyrosine $-1,01$ times $(\mathrm{p} \leq 0,001)$ compared to control parameters.

However, in pork treated with a solution of hydrogen peroxide increased the content of arginine $-6,38$ times $(\mathrm{p} \leq 0,001)$, O-Proline $-2,38$ times $(\mathrm{p} \leq 0,001)$, aspartic acid and valine $-1,18$ times $(\mathrm{p} \leq 0,001)$, methionine by 1,14 times $(p \leq 0,001)$, threonine - by 1,11 times $(p \leq 0,001)$, and other amino acids (proline, lysine, serine, phenylalanine, isoleucine) by the quantitative content slightly increased, respectively $-1,09,1,07,1,05,1,04$ times ( $\mathrm{p} \leq 0,001)$ compared to the control indicators.

When treating pork of dubious freshness with alkaline detergents, the total amino acid content was probably reduced by 1,02 times $(p \leq 0,001)$ compared to the parameters of fresh pork, namely by reducing alanine by 1,45 times $(p \leq 0,001)$ acids $-1,14$ times $(p \leq 0,001)$, glycine $-1,13$ times $(\mathrm{p} \leq 0,001)$, histidine $-1,09$ times $(\mathrm{p} \leq 0,001)$, leucine $-1,05(\mathrm{p} \leq 0,001)$, as well as lysine and aspartic acid $-1,03$ times $(p \leq 0,001)$. At the same time, the quantitative content of individual amino acids probably increased: arginine $-6,39$ times $(\mathrm{p} \leq 0,001)$, O-proline $-1,71$ times $(\mathrm{p} \leq 0,001)$, methionine $-1,31$ times $(\mathrm{p} \leq 0,001)$, valine $-1,30$ times $(\mathrm{p} \leq 0,001)$, proline $-1,25$ times $(\mathrm{p} \leq 0,001)$, cystine $-1,24$ times $(\mathrm{p} \leq 0,001)$, threonine $-1,18$ times $(p \leq 0,001)$, serine $-1,14$ times $(p \leq 0,001)$. And the quantitative content of such amino acids - tyrosine and isoleucine and phenylalanine, respectively, increased slightly - by $1,6(\mathrm{p} \leq 0,001)$ and 1,11 times $(\mathrm{p} \leq 0,001)$ compared to the control indicators.

In table 4 summarizes the content scor in pork of different quality. 
The content scor in amino acids $(\%), M \pm m, n=36$

\begin{tabular}{|c|c|c|c|c|}
\hline $\begin{array}{c}\text { Amino } \\
\text { acids }\end{array}$ & $\begin{array}{c}\text { Fresh pork } \\
\text { (control) }\end{array}$ & $\begin{array}{c}\text { Pork of } \\
\text { dubious } \\
\text { freshness }\end{array}$ & $\begin{array}{c}\text { Pork treated with } \\
\text { hydrogen peroxide } \\
\mathbf{5 \%}\end{array}$ & $\begin{array}{c}\text { The pork is } \\
\text { treated with } \\
\text { alkaline } \\
\text { detergents and } \\
\text { disinfectants }\end{array}$ \\
\hline Lysine & $166,00 \pm 0,38$ & $\begin{array}{c}146,00 \pm \\
0,47 * * *\end{array}$ & $179,00 \pm 0,34^{* * *}$ & $163,00 \pm 0,71^{* * *}$ \\
\hline Threonine & $114,00 \pm 0,38$ & $115,00 \pm 0,32$ & $128,00 \pm 0,65^{* * *}$ & $137,00 \pm 0,75^{* * *}$ \\
\hline Cystine & $100,00 \pm 0,75$ & $\begin{array}{c}133,00 \pm \\
0,51^{* * *}\end{array}$ & $114,00 \pm 0,47^{* * *}$ & $131,00 \pm 0,49^{* * *}$ \\
\hline Valin & $86,00 \pm 0,42$ & $\begin{array}{c}106,00 \pm \\
0,56 * * *\end{array}$ & $103,00 \pm 0,78^{* * *}$ & $114,00 \pm 0,31^{* * *}$ \\
\hline Isoleucine & $117,00 \pm 0,43$ & $\begin{array}{c}137,00 \pm \\
0,71 * * *\end{array}$ & $124,00 \pm 0,77^{* * *}$ & $132,00 \pm 0,51^{* * *}$ \\
\hline Leucine & $141,00 \pm 0,54$ & $\begin{array}{c}127,00 \pm \\
0,49 * * *\end{array}$ & $126,00 \pm 0,49 * * *$ & $136,00 \pm 0,49 *$ \\
\hline Tyrosine & $134,00 \pm 0,41$ & $\begin{array}{c}168,00 \pm \\
0,33^{* * *}\end{array}$ & $138,00 \pm 0,79 * * *$ & $148,00 \pm 0,63^{* * *}$ \\
\hline
\end{tabular}

Note: $*-p<0,05 ; * * *-p<0,001$.

The lysine scor in pork of questionable freshness and pork treated with alkaline detergents was significantly reduced respectively - by 1,14 times ( $p$ $\leq 0,001)$ and 1,02 times $(\mathrm{p} \leq 0,001)$, and in pork treated with hydrogen peroxide solution $(5 \%)$ probably increased by 1,08 times $(\mathrm{p} \leq 0,001)$ compared to fresh pork. The threonine content scorof pork of questionable freshness, doubtful freshness when treated with a solution of hydrogen peroxide $(5 \%)$ and alkaline detergents significantly increased, respectively 1,01 times, 1,12 times $(\mathrm{p} \leq 0,001)$ and 1,20 times $(\mathrm{p} \leq 0,001)$; the cystine scor is 1,33 times $(p \leq 0,001), 1,14$ times $(p \leq 0,001)$ and 1,31 times, respectively ( $\mathrm{p} \leq 0,001)$; the valine scor, respectively, is 1,23 times $(\mathrm{p} \leq 0,001), 1,20$ times $(\mathrm{p} \leq 0,001)$ and 1,33 times $(\mathrm{p} \leq 0,001)$; the isoleucine scor, respectively, is 1,17 times $(\mathrm{p} \leq 0,001), 1,06(\mathrm{p} \leq 0,001)$ and 1,13 times $(p \leq 0,001)$; the tyrosine rate, respectively, was 1,25 times $(p \leq 0,001), 1,03$ times $(p \leq 0,001)$ and 1,10 times $(p \leq 0,001)$. However, the leucine score in pork of questionable freshness, pork treated with a solution of hydrogen peroxide (5\%) and treated with alkaline detergents significantly decreased, respectively - by 1,11 times $(p \leq 0,001), 1,12$ times $(p \leq 0,001)$ and 1,04 times $(\mathrm{p} \leq 0,05)$.

\section{Establishment of amino acid composition in mutton and meat of goat for their treatment with detergents}

Analysis of the amino acids composition of mutton of different quality is presented in table 5 . 
Table 5

Amino acids composition of mutton of different quality, $\mathbf{M} \pm \mathbf{m}, \mathbf{n}=\mathbf{2 7}$

\begin{tabular}{|c|c|c|c|c|c|c|}
\hline \multirow{3}{*}{ Amino acids } & \multicolumn{6}{|c|}{ Mutton of different quality } \\
\hline & \multicolumn{2}{|c|}{$\begin{array}{l}\text { Fresh mutton } \\
\quad \text { (control) }\end{array}$} & \multicolumn{2}{|c|}{$\begin{array}{l}\text { Mutton of dubious } \\
\text { freshness }\end{array}$} & \multicolumn{2}{|c|}{$\begin{array}{l}\text { Mutton of dubious } \\
\text { freshness when } \\
\text { treated with } \\
\text { potassium } \\
\text { permanganate }(5 \%)\end{array}$} \\
\hline & $\begin{array}{c}\text { quantity, } \\
\text { mg }\end{array}$ & $\begin{array}{c}\text { \% by } \\
\text { mg }\end{array}$ & $\begin{array}{c}\text { quantity, } \\
\text { mg } \\
(* * *)\end{array}$ & $\begin{array}{c}\% \text { by } \\
\text { mg }\end{array}$ & $\underset{\text { mg }}{\text { quantity, }}$ & $\begin{array}{c}\% \text { by } \\
\text { mg }\end{array}$ \\
\hline Lysine & $\begin{array}{c}1,707 \pm \\
0,0009\end{array}$ & 8,21 & $\begin{array}{l}1,656 \pm \\
0,0037\end{array}$ & 8,01 & $\begin{array}{l}1,975 \pm \\
0,0019\end{array}$ & 10,39 \\
\hline Histidine & $\begin{array}{c}0,502 \pm \\
0,0019\end{array}$ & 2,41 & $\begin{array}{c}0,729 \pm \\
0,0050\end{array}$ & 3,53 & $\begin{array}{l}0,613 \pm \\
0,0028\end{array}$ & 3,22 \\
\hline Arginine & $\begin{array}{l}1,127 \pm \\
0,0011\end{array}$ & 5,42 & $\begin{array}{l}1,131 \pm \\
0,0021\end{array}$ & 5,47 & $\begin{array}{l}1,148 \pm \\
0,0022\end{array}$ & 6,04 \\
\hline O-Proline & $\begin{array}{c}0,323 \pm \\
0,0006\end{array}$ & 1,55 & $\begin{array}{c}0,292 \pm \\
0,0066\end{array}$ & 1,41 & $\begin{array}{c}0,162 \pm \\
0,0032\end{array}$ & 0,85 \\
\hline Aspartic acid & $\begin{array}{l}1,627 \pm \\
0,0009\end{array}$ & 7,83 & $\begin{array}{l}1,316 \pm \\
0,0025\end{array}$ & 6,36 & $\begin{array}{l}1,360 \pm \\
0,0031\end{array}$ & 7,16 \\
\hline Threonine & $\begin{array}{c}0,922 \pm \\
0,0006\end{array}$ & 4,43 & $\begin{array}{c}0,959 \pm \\
0,0077\end{array}$ & 4,64 & $\begin{array}{c}0,846 \pm \\
0,0021\end{array}$ & 4,45 \\
\hline Serine & $\begin{array}{c}0,829 \pm \\
0,0003\end{array}$ & 3,99 & $\begin{array}{c}0,834 \pm \\
0,0021\end{array}$ & 4,03 & $\begin{array}{c}0,728 \pm \\
0,0022\end{array}$ & 3,83 \\
\hline Glutamic acid & $\begin{array}{c}3,679 \pm \\
0,0513\end{array}$ & 17,70 & $\begin{array}{c}3,395 \pm \\
0,0043\end{array}$ & 16,42 & $\begin{array}{c}3,491 \pm \\
0,0011\end{array}$ & 18,37 \\
\hline Proline & $\begin{array}{l}1,170 \pm \\
0,0069\end{array}$ & 5,63 & $\begin{array}{l}1,140 \pm \\
0,0019\end{array}$ & 5,51 & $\begin{array}{c}0,805 \pm \\
0,0014\end{array}$ & 4,24 \\
\hline Glycine & $\begin{array}{c}1,350 \pm \\
0,0081\end{array}$ & 6,49 & $\begin{array}{l}1,170 \pm \\
0,0023\end{array}$ & 5,66 & $\begin{array}{l}1,056 \pm \\
0,0018\end{array}$ & 5,56 \\
\hline Alanine & $\begin{array}{l}1,808 \pm \\
0,0011\end{array}$ & 8,70 & $\begin{array}{l}1,479 \pm \\
0,0046\end{array}$ & 7,15 & $\begin{array}{l}1,072 \pm \\
0,0017\end{array}$ & 5,64 \\
\hline Cystine & $\begin{array}{c}0,192 \pm \\
0,0013\end{array}$ & 0,92 & $\begin{array}{c}0,156 \pm \\
0,0016\end{array}$ & 0,75 & $\begin{array}{l}1,108 \pm \\
0,0013\end{array}$ & 0,57 \\
\hline Valine & $\begin{array}{c}0,866 \pm \\
0,0012\end{array}$ & 4,17 & $\begin{array}{l}1,038 \pm \\
0,0023\end{array}$ & 5,02 & $\begin{array}{c}0,866 \pm \\
0,0012\end{array}$ & 4,56 \\
\hline Methionine & $\begin{array}{c}0,499 \pm \\
0,0018\end{array}$ & 2,40 & $\begin{array}{c}0,623 \pm \\
0,0048\end{array}$ & 3,01 & $\begin{array}{l}0,596 \pm \\
0,0029\end{array}$ & 3,14 \\
\hline Isoleucine & $\begin{array}{c}0,876 \pm \\
0,0019\end{array}$ & 4,21 & $\begin{array}{l}1,022 \pm \\
0,0034\end{array}$ & 4,94 & $\begin{array}{c}0,865 \pm \\
0,0019\end{array}$ & 4,55 \\
\hline Leucine & $\begin{array}{l}1,800 \pm \\
0,0019\end{array}$ & 8,66 & $\begin{array}{l}1,812 \pm \\
0,0040\end{array}$ & 8,76 & $\begin{array}{l}1,787 \pm \\
0,0026\end{array}$ & 9,41 \\
\hline Tyrosine & $\begin{array}{c}0,692 \pm \\
0,0018\end{array}$ & 3,33 & $\begin{array}{c}0,888 \pm \\
0,0028\end{array}$ & 4,29 & $\begin{array}{c}0,678 \pm \\
0,0060\end{array}$ & 3,57 \\
\hline Phenylalanine & $\begin{array}{c}0,818 \pm \\
0,0015\end{array}$ & 3,94 & $\begin{array}{l}1,040 \pm \\
0,0026\end{array}$ & 5,03 & $\begin{array}{c}0,845 \pm \\
0,0033\end{array}$ & 4,45 \\
\hline $\begin{array}{c}\text { Summary } \\
\text { content }\end{array}$ & $\begin{array}{c}20,786 \pm \\
0,0430\end{array}$ & 100,0 & $\begin{array}{l}20,679 \pm \\
0,0440 *\end{array}$ & 100,0 & $\begin{array}{c}19,000 \pm \\
0,0480 * * *\end{array}$ & 100,0 \\
\hline
\end{tabular}

Note: $*-p \leq 0,05 ; * * *-p \leq 0,001-$ for all amino acids by the quantitative content in $\mathrm{mg}$ of mutton of doubtful freshness and potassium permanganate treated. 
The total amino acid content of fresh lamb was $20,786 \pm 0,043 \mathrm{mg}$ $(100 \% \mathrm{mg})$ and of lamb of doubtful freshness was 1,005 times $(\mathrm{p} \leq 0,05)$, which was $0,107 \mathrm{mg}$ less than that of fresh mutton. This is justified by the fact that in the mutton of doubtful freshness a significant decrease in the quantitative content of aspartic acid was found - by 1,24 times $(p \leq 0.001)$; cystine $-1,23$ times $(\mathrm{p} \leq 0,001)$; alanine $-1,22$ times $(\mathrm{p} \leq 0,001)$; glycine 1,15 times $(\mathrm{p} \leq 0,001)$; O-Proline $-1,11$ times $(\mathrm{p} \leq 0,001)$; lysine and proline $-1,03$ times $(p \leq 0,001)$. However, at the same time, in the mutton of doubtful freshness, there was a probable increase in the content of such amino acids as: histidine - by 1,28 times $(\mathrm{p} \leq 0,001)$; tyrosine $-1,28$ times $(\mathrm{p} \leq 0,001)$; phenylalanine $-1,27$ times $(\mathrm{p} \leq 0,001)$; methionine $-1,25$ times $(\mathrm{p} \leq 0,001)$; valine $-1,20$ times $(\mathrm{p}<0,001)$; isoleucine $-1,17$ times $(\mathrm{p} \leq 0,001)$, threonine $-1,04$ times $(\mathrm{p} \leq 0,001)$. There is no significant difference in arginine and serine content.

Significant reduction of total amino acid content of mutton of doubtful freshness by treatment with potassium permanganate solution $(5 \%)$ was found to be 1,79 times $(\mathrm{p} \leq 0,001)$ compared to parameters of fresh mutton. Namely, the quantitative content of amino acids significantly decreased compared to the control indicators: O-Proline $-1,99$ times $(p \leq 0,001)$; cystine $-1,75$ times $(p \leq 0,001)$; alanine $-1,69$ times $(p \leq 0,001)$; Proline 1,45 times $(\mathrm{p} \leq 0,001)$; aspartic acid $-1,20$ times $(\mathrm{p} \leq 0,001)$; threonine 1,17 times $(\mathrm{p} \leq 0,001)$; serine $-1,14$ times $(\mathrm{p} \leq 0,001)$, glutamic acid 1,05 times $(\mathrm{p} \leq 0,001)$. The content of isoleucine, leucine, tyrosine decreased slightly, and the difference in indicators was significant.

The content of other amino acids in the treatment of lamb with a solution of potassium permanganate significantly increased: histidine - by 1,22 times ( $\mathrm{p} \leq 0,001)$; methionine $-1,19$ times $(\mathrm{p} \leq 0,001)$; lysine $-1,16$ times $(\mathrm{p} \leq 0,001)$; and arginine $-1,02$ times $(\mathrm{p} \leq 0,001)$ and phenylalanine $-1,03$ times $(p \leq 0,001)$ compared to the control parameters. There is no significant difference in the valine content.

Table 6 summarizes the content scor of feeds in mutton of different quality.

Table 6

The content scor in amino acids $(\%), \mathbf{M} \pm \mathbf{m}, \mathbf{n}=\mathbf{2 7}$

\begin{tabular}{|c|c|c|c|}
\hline Amino acids & $\begin{array}{c}\text { Fresh mutton } \\
\text { (control) }\end{array}$ & $\begin{array}{c}\text { Mutton of dubious } \\
\text { freshness }\end{array}$ & $\begin{array}{c}\text { Mutton of dubious freshness } \\
\text { when treated with potassium } \\
\text { permanganate (5\%) }\end{array}$ \\
\hline Lysine & $149,00 \pm 0,36$ & $146,00 \pm 0,37^{* * *}$ & $189,00 \pm 0,20^{* * * *}$ \\
\hline Threonine & $111,00 \pm 0,32$ & $116,00 \pm 0,31^{* * *}$ & $111,00 \pm 0,21^{* * *}$ \\
\hline Cystine & $95,00 \pm 0,47$ & $108,00 \pm 0,25^{* * *}$ & $106,00 \pm 0,23^{* * *}$ \\
\hline Valin & $83,00 \pm 0,38$ & $100,00 \pm 0,97^{* * *}$ & $91,00 \pm 0,23^{* * *}$ \\
\hline Isoleucine & $105,00 \pm 0,39$ & $124,00 \pm 0,27^{* * *}$ & $114,00 \pm 0,21^{* * *}$ \\
\hline Leucine & $124,00 \pm 0,41$ & $125,00 \pm 0,24^{*}$ & $134,00 \pm 0,33^{* * *}$ \\
\hline Tyrosine & $121,00 \pm 0,38$ & $155,00 \pm 0,82^{* * *}$ & $134,00 \pm 0,60^{* * *}$ \\
\hline
\end{tabular}

Note: $*-p \leq 0,05 ; * * *-p \leq 0,001$. 
The lysine scor in lambs of questionable freshness was probably decreased by 1,02 times ( $\mathrm{p} \leq 0,001)$, and in muttons by treatment with potassium permanganate solution - it was significantly increased by 1,27 times $(\mathrm{p} \leq 0,001)$ compared to the parameters in fresh mutton. The threonine content scor of mutton of doubtful freshness probably increased by 1,05 times $(\mathrm{p} \leq 0,001)$, and in mutton treated with potassium permanganate solution this index was unchanged $(111,00 \%)$. The cystine scor in mutton of questionable freshness and mutton processed significantly increased, respectively, by 1,14 times $(p \leq 0,001)$ and 1,12 times $(p<0,001)$; the valine scor is 1,20 times $(p \leq 0,001)$ and 1,10 times $(p \leq 0,001)$; the isoleucine scor is 1,18 times $(\mathrm{p} \leq 0,001)$ and 1,09 times $(\mathrm{p} \leq 0,001)$; the leucine scor is 1,01 times $(p \leq 0,05)$ and 1.08 times $(p \leq 0,001)$; the tyrosine scor is 1,28 times $(\mathrm{p} \leq 0,001)$ and 1,11 times $(\mathrm{p} \leq 0,001)$.

Analysis of the amino acid composition of the meat of goat of different quality is presented in table 7 .

Table 7

Amino acid composition of meat of goat of different quality, $\mathbf{M} \pm \mathbf{m}, \mathbf{n}=\mathbf{2 7}$

\begin{tabular}{|c|c|c|c|c|c|c|}
\hline \multirow{3}{*}{ Amino acids } & \multicolumn{6}{|c|}{ Meat of goat of different quality } \\
\hline & \multicolumn{2}{|c|}{$\begin{array}{c}\text { Fresh meat of goat } \\
\text { (control) }\end{array}$} & \multicolumn{2}{|c|}{$\begin{array}{c}\text { Meat of goat of } \\
\text { dubious freshness }\end{array}$} & \multicolumn{2}{|c|}{$\begin{array}{c}\text { Meat of goat of } \\
\text { dubious freshness by } \\
\text { acetic acid treatment } \\
(10 \%)\end{array}$} \\
\hline & $\begin{array}{c}\text { quantity, } \\
\text { mg }\end{array}$ & $\begin{array}{c}\text { \% by } \\
\text { mg }\end{array}$ & $\begin{array}{l}\text { quantity, } \\
\text { mg (***) }\end{array}$ & $\begin{array}{c}\text { \% by } \\
\text { mg }\end{array}$ & $\begin{array}{c}\text { quantity, mg } \\
(* * *)\end{array}$ & $\begin{array}{c}\text { \% by } \\
\text { mg }\end{array}$ \\
\hline 1 & 2 & 3 & 4 & 5 & 6 & 7 \\
\hline Lysine & $\begin{array}{l}1,907 \pm \\
0,0024\end{array}$ & 9,19 & $\begin{array}{l}1,621 \pm \\
0,0023\end{array}$ & 7,85 & $\begin{array}{c}2,114 \pm \\
0,0039\end{array}$ & 10,35 \\
\hline Histidine & $\begin{array}{c}0,553 \pm \\
0,0032\end{array}$ & 2,67 & $\begin{array}{c}0,665 \pm \\
0,0062\end{array}$ & 3,22 & $\begin{array}{c}0,611 \pm \\
0,0042\end{array}$ & 2,99 \\
\hline Arginine & $\begin{array}{c}0,943 \pm \\
0,0040\end{array}$ & 4,54 & $\begin{array}{l}1,028 \pm \\
0,0024\end{array}$ & 4,98 & $\begin{array}{c}0,971 \pm \\
0,0027\end{array}$ & 4,76 \\
\hline O-Proline & $\begin{array}{c}0,128 \pm \\
0,0017\end{array}$ & 0,62 & $\begin{array}{c}0,221 \pm \\
0,0040\end{array}$ & 1,07 & $\begin{array}{l}0,135 \pm \\
0,0017\end{array}$ & 0,66 \\
\hline Aspartic acid & $\begin{array}{l}1,679 \pm \\
0,0052\end{array}$ & 8,09 & $\begin{array}{l}1,519 \pm \\
0,0015 \\
\end{array}$ & 7,35 & $\begin{array}{l}1,570 \pm \\
0,0027\end{array}$ & 7,69 \\
\hline Threonine & $\begin{array}{c}0,989 \pm \\
0,0018\end{array}$ & 4,76 & $\begin{array}{l}0,992 \pm \\
0,0022\end{array}$ & 4,80 & $\begin{array}{c}0,953 \pm \\
0,0027\end{array}$ & 4,67 \\
\hline Serine & $\begin{array}{l}0,881 \pm \\
0,0019\end{array}$ & 4,24 & $\begin{array}{c}0,843 \pm \\
0,0039\end{array}$ & 4,08 & $\begin{array}{l}0,873 \pm \\
0,0021\end{array}$ & 4,27 \\
\hline Glutamic acid & $\begin{array}{c}3,800 \pm \\
0,0037 \\
\end{array}$ & 18,30 & $\begin{array}{c}3,582 \pm \\
0,0039 \\
\end{array}$ & 17,34 & $\begin{array}{c}3,641 \pm \\
0,0031 \\
\end{array}$ & 17,83 \\
\hline Proline & $\begin{array}{c}0,821 \pm \\
0,0020\end{array}$ & 3,96 & $\begin{array}{c}0,944 \pm \\
0,0033\end{array}$ & 4,57 & $\begin{array}{c}0,798 \pm \\
0,0023\end{array}$ & 3,91 \\
\hline Glycine & $\begin{array}{l}1,128 \pm \\
0,0039\end{array}$ & 5,44 & $\begin{array}{l}1,103 \pm \\
0,0027\end{array}$ & 5,34 & $\begin{array}{l}1,158 \pm \\
0,0019\end{array}$ & 5,67 \\
\hline Alanine & $\begin{array}{l}1,381 \pm \\
0,0066\end{array}$ & 6,65 & $\begin{array}{l}1,380 \pm \\
0,0030\end{array}$ & 6,68 & $\begin{array}{l}1,320 \pm \\
0,0030\end{array}$ & 6,47 \\
\hline
\end{tabular}


Continuation of Table 7

\begin{tabular}{|c|c|c|c|c|c|c|}
\hline $\mathbf{1}$ & $\mathbf{2}$ & $\mathbf{3}$ & $\mathbf{4}$ & $\mathbf{5}$ & $\mathbf{6}$ & $\mathbf{7}$ \\
\hline Cystine & $\begin{array}{c}0,192 \pm \\
0,0021\end{array}$ & 0,92 & $\begin{array}{c}0,197 \pm \\
0,0018\end{array}$ & 0,95 & $\begin{array}{c}0,181 \pm \\
0,0031\end{array}$ & 0,89 \\
\hline Valine & $\begin{array}{c}0,944 \pm \\
0,0018\end{array}$ & 4,55 & $\begin{array}{c}1,010 \pm \\
0,0026\end{array}$ & 4,89 & $\begin{array}{c}1,029 \pm \\
0,0018\end{array}$ & 5,04 \\
\hline Methionine & $\begin{array}{c}0,577 \pm \\
0,0006\end{array}$ & 2,78 & $\begin{array}{c}0,608 \pm \\
0,0029\end{array}$ & 2,94 & $\begin{array}{c}0,607 \pm \\
0,0027\end{array}$ & 2,97 \\
\hline Isoleucine & $\begin{array}{c}0,966 \pm \\
0,0010\end{array}$ & 4,65 & $\begin{array}{c}1,081 \pm \\
0,0033\end{array}$ & 4,23 & $\begin{array}{c}0,919 \pm \\
0,0017\end{array}$ & 4,50 \\
\hline Leucine & $\begin{array}{c}2,140 \pm \\
0,0042\end{array}$ & 19,31 & $\begin{array}{c}1,905 \pm \\
0,0040\end{array}$ & 9,22 & $\begin{array}{c}1,847 \pm \\
0,0021\end{array}$ & 9,04 \\
\hline Tyrosine & $\begin{array}{c}0,817 \pm \\
0,0011\end{array}$ & 3,94 & $\begin{array}{c}0,918 \pm \\
0,0032\end{array}$ & 4,45 & $\begin{array}{c}0,774 \pm \\
0,0033\end{array}$ & 3,79 \\
\hline Phenylalanine & $\begin{array}{c}0,915 \pm \\
0,0009\end{array}$ & 4,41 & $\begin{array}{c}1,044 \pm \\
0,0028\end{array}$ & 5,05 & $\begin{array}{c}0,919 \pm \\
0,0031\end{array}$ & 4,50 \\
\hline Summary & $20,762 \pm$ \\
content & 0,0440 & 100,0 & $\begin{array}{c}20,660 \pm \\
0,0710\end{array}$ & $\begin{array}{c}100, \\
0\end{array}$ & $\begin{array}{c}20,420 \pm \\
0,0660^{* * *}\end{array}$ & 100,0 \\
\hline
\end{tabular}

Note: $* * *-p \leq 0,001-$ for all amino acids by the quantitative content in $m g$ in goat of questionable freshness and treated with acetic acid solution.

In meat of goat of doubtful freshness, the total amino acid content was $20,660 \pm 0,071 \mathrm{mg}$, which is 1,005 times less than that of fresh meat of goat. The decrease in the total content of amino acids in the meat of goat of doubtful degree of freshness was characterized by a decrease in the quantitative content of such amino acids as: lysine - by 1,18 times $(\mathrm{p} \leq 0,001)$; leucine $-1,12$ times $(\mathrm{p} \leq 0,001)$; aspartic acid $-1,11$ times $(\mathrm{p} \leq 0,001)$; glutamic acid $-1,06$ times $(\mathrm{p} \leq 0,001)$; serine $-1,05$ times $(\mathrm{p} \leq 0,001)$; glycine $-1,02$ times $(\mathrm{p} \leq 0,001)$. Along with this, there was an increase in the quantitative content of such amino acids compared to indicators of control, such as: O-Proline $-1,73$ times $(\mathrm{p} \leq 0,001)$; histidine 1,20 times $(\mathrm{p} \leq 0,001)$; proline $-1,15$ times $(\mathrm{p} \leq 0,001)$; phenylalanine 1,14 times $(\mathrm{p} \leq 0,001)$; isoleucine and tyrosine $-1,12$ times $(\mathrm{p} \leq 0,001)$; arginine $-1,09$ times $(\mathrm{p} \leq 0,001)$; as well as a slight significant increase $(\mathrm{p} \leq 0,001)$ in the quantitative content of amino acids (valine, methionine and cystine) by $1,07,1,05$ and 1,03 times, respectively. There is no significant difference in the content of alanine and threonine.

When treating the meat of goat of doubtful freshness with acetic acid with a mass concentration of $10 \%$, the total amino acid content was $20,420 \pm$ $0,066 \mathrm{mg}$, which is 1,011 times $(\mathrm{p} \leq 0,001)$ less than that of fresh meat of goat (control). Analysis of the content of individual amino acids was characterized by a significant decrease in the quantitative content of leucine $-1,16$ times $(\mathrm{p}<0,001)$; aspartic acid $-1,07$ times $(\mathrm{p} \leq 0,001)$; cystine, tyrosine - 1,06 times ( $\mathrm{p} \leq 0,001)$; alanine and isoleucine 1,05 times $(\mathrm{p} \leq 0,001)$; threonine and glutamic acid $-1,04$ times $(\mathrm{p}<0,001)$; 
proline $-1,03$ times $(\mathrm{p} \leq 0,001)$; serine is 1,01 times $(\mathrm{p} \leq 0,001)$ compared to control values.

A significant slight increase in lysine content was also found to be 1.11 times $(p \leq 0,001)$; histidine $-1,10$ times $(p \leq 0,001)$; valine $-1,09$ times $(\mathrm{p} \leq 0,001)$; methionine, O-proline $-1,05$ times $(\mathrm{p} \leq 0,001)$; arginine, glycine $-1,03$ times $(p \leq 0,001)$. There is no significant difference in phenylalanine content.

Table 8 summarizes the content scor of meat of goats in different goats.

Table 8

The content scor in amino acids $(\%), \mathbf{M} \pm \mathbf{m}, \mathbf{n}=\mathbf{2 7}$

\begin{tabular}{|c|c|c|c|}
\hline Amino acids & $\begin{array}{c}\text { Fresh meat of } \\
\text { goat (control) }\end{array}$ & $\begin{array}{c}\text { Meat of goat of } \\
\text { dubious freshness }\end{array}$ & $\begin{array}{c}\text { Meat of goat of dubious } \\
\text { freshness by acetic acid } \\
\text { treatment (10\%) }\end{array}$ \\
\hline Lysine & $167,00 \pm 0,38$ & $143,00 \pm 0,33^{* * *}$ & $188,00 \pm 0,53^{* * *}$ \\
\hline Threonine & $119,00 \pm 0,42$ & $120,00 \pm 0,36$ & $117,00 \pm 0,21^{* *}$ \\
\hline Cystine & $106,00 \pm 0,36$ & $111,00 \pm 0,40^{* * *}$ & $110,00 \pm 0,44^{* * *}$ \\
\hline Valin & $91,00 \pm 0,45$ & $98,00 \pm 0,41^{* *}$ & $101,00 \pm 0,41^{* * *}$ \\
\hline Isoleucine & $116,00 \pm 0,43$ & $131,00 \pm 0,38^{* * *}$ & $112,00 \pm 0,43^{* * *}$ \\
\hline Leucine & $147,00 \pm 0,41$ & $132,00 \pm 0,34^{*}$ & $139,00 \pm 0,49^{* * *}$ \\
\hline Tyrosine & $139,00 \pm 0,39$ & $158,00 \pm 0,36^{* * *}$ & $138,00 \pm 0,52$ \\
\hline
\end{tabular}

Note: $* *-p<0,01 ; * * *-p<0,001$.

The lysine score in fresh meat of goat was $167,00 \pm 0,38 \%$, and in the goat of questionable freshness, this indicator decreased significantly by 1,17 times $(p<0,001)$ compared to that of fresh goat. The threonine scor of meat of goat of doubtful freshness increased slightly by 1.01 times; cystinescor was 1,05 -fold ( $\mathrm{p}<0,001$ ); the valinescor was 1,08 times $(\mathrm{p}<0,001)$; the isoleucine scor was 1,13 times $(\mathrm{p}<0,001)$ and the tyrosine score was 1,14 times $(\mathrm{p}<0,001)$. However, the leucine scor was likely to decrease 1,11 times $(\mathrm{p}<0,001)$ and lysine $-1,17$ times $(\mathrm{p}<0,001)$.

And for the treatment of meat of goat with a solution of acetic acid $(10 \%)$, the lysine scor increased significantly - by 1,13 times $(\mathrm{p}<0,001)$, and threonine decreased significantly - by 1,02 times ( $p<0,001)$; the cystine and valine scor increased slightly, respectively, at $1,04(\mathrm{p}<0,001)$ and 1,11 times $(\mathrm{p}<0,001)$; the isoleucine, leucine, and tyrosine scor were likely to decrease $1,04(\mathrm{p} \leq 0,001), 1,04(\mathrm{p}<0,001)$, and 1,01, respectively.

It should be noted that in practice, the most widely used to determine biological value was the so-called methods of amino acid scales, which are based on the use of the amino acid (chemical) score - the integrated amino acid index of the Kunau-Auxerre-Mitchell and the Korpachi index. Amino acid score allows to identify the limiting essential amino acids and the degree of their deficiency that is relative to the percentage of amino acids in the investigated protein and in the same amount of conditioned "ideal" 
protein, which completely satisfies the needs of the body ${ }^{20}$. All amino acids in which the crunch is less than $100 \%$ are considered to be limiting, and the lowest acidic amino acid is the main limiting $\operatorname{acid}^{21}$.

Limiting amino acids were established in fresh beef - valine (score $83,00 \pm 0,42 \%)$, in formalin-treated beef cystine $(92,00 \pm 0,830$ and tyrosine $(88,00 \pm 0,49)$.

The limiting amino acid of valine was established in pork fresh and treated with a solution of hydrogen peroxide, respectively $-86,00 \pm 0,42 \%$ and $103,00 \pm 0,78 \%$. Limiting amino acids in mutton fresh: cystine $-95,00 \pm 0,47 \%$ and valine $-83,00 \pm 0,38 \%$; in mutton of doubtful freshness: valine $100,00 \pm 0,97 \%$, cystine $-108,00 \pm 0,25 \%$; in meat of lamb treated with potassium permanganate solution: valine $-91,00 \pm 0,23 \%$, cystine $-106,00 \pm$ $0,23 \%$. Limiting amino acids in meat of goat fresh: valine $-91,00 \pm 0,45 \%$; in goat of doubtful freshness: valine $-98,00 \pm 0,41 \%$, in meat of goat treated with potassium permanganate solution: valine $-101,00 \pm 0,41 \%$.

\section{CONCLUSIONS}

The total content of essential amino acids was slightly increased in beef of doubtful freshness by $0,019 \mathrm{mg}$ and treated with chlorine solution (activity $3 \%$ ) by $0,102 \mathrm{mg}$, and in beef treated with formalin solution (10\%) was slightly reduced $(1,10$ times $) \mathrm{p} \leq 0,001)$; and the content of substitutable amino acids significantly decreased in beef of dubious freshness by 1,04 times $(\mathrm{p} \leq 0,001)$, beef treated with formalin solution 1,27 times $(\mathrm{p} \leq 0,001)$, beef treated with chlorine solution $-1,12$ times $(\mathrm{p} \leq 0,001)$ compared to controls. The total content of essential amino acids in pork of dubious freshness was slightly increased by $0,008 \mathrm{mg}$, in pork treated with hydrogen peroxide $(5 \%)$ by $0,295 \mathrm{mg}$, which is 1,04 times higher $(\mathrm{p} \leq$ 0,001 ), and in pork treated with alkaline detergent-disinfectant $0,645 \mathrm{mg}$, which is 1,08 times more $(\mathrm{p} \leq 0,001)$ compared to control indicators; and the content of substitutable amino acids in pork of dubious freshness and pork treated with hydrogen peroxide solution and alkaline detergents was significantly reduced respectively by 1,08 times, 1,04 and 1,08 times $(\mathrm{p} \leq$ $0,001)$.The total content of essential amino acids in mutton of dubious freshness was significantly increased by $0,662 \mathrm{mg}$, which is 1,09 times more $(\mathrm{p} \leq 0,001)$, in mutton treated with potassium permanganate solution $(5 \%)$ by $0,292 \mathrm{mg}$, which is 1.04 times more $(\mathrm{p} \leq 0,001)$ compared to control indicators; and the content of substitutable amino acids was significantly reduced by $0,769 \mathrm{mg}$ and $2,078 \mathrm{mg}$ in accordance with the quality of mutton, which is 1,06 and 1,19 times less $(p \leq 0,001)$ compared to the control indicators. The total content of essential amino acids in the meat of goat of dubious freshness was significantly reduced by $0,177 \mathrm{mg}$, which is

20 Tsehmistrenko S., Tsehmistrenko O. The Biochemistry of Meat and Meat Products. Bila Tserkva, 2014. 192 p.

21 Dudchik N. A new method for determining the relative biological value of a protein component of food. Nutrition Problems. 2006. № 4. P. 4-6. 
1,03 times less $(\mathrm{p} \leq 0,001)$, in meat of goat treated with acetic acid solution $(10 \%)$ by $0,050 \mathrm{mg}$, which is 1,01 times less $(\mathrm{p} \leq 0,001)$ compared to control indicators; and the content of substitutable amino acids in the meat of goat of dubious freshness was slightly increased significantly by $0,075 \mathrm{mg}$, which is 1,01 times higher $(p \leq 0,001)$, and in meat of goat treated with acetic acid was reduced by $0,292 \mathrm{mg}$, which is 1,02 times less $(\mathrm{p} \leq 0,001)$ compared to controls.

\section{SUMMARY}

In order to obtain safe food products, it is necessary to carry out riskoriented monitoring of the detection of detergents in slaughtered animals for the production and handling due to the breach of conditions and timing when using patented express methods. Studies have found the amino acid composition of the meat of slaughtered animals of various qualities, including for the treatment of detergents. The total content of essential amino acids was slightly increased in beef of doubtful freshness by $0,019 \mathrm{mg}$ and treated with chlorine solution (activity $3 \%$ ) by $0,102 \mathrm{mg}$, and in beef treated with formalin solution (10\%) was slightly reduced by 1,10 times $(\mathrm{p} \leq$ $0,001)$; and the content of replacement amino acids significantly decreased in beef of dubious freshness by 1,04 times $(p \leq 0,001)$, beef treated with formalin solution 1,27 times $(\mathrm{p} \leq 0,001)$, beef treated with chlorine solution $-1,12$ times $(p \leq 0,001)$ compared to controls. The total content of essential amino acids in pork of dubious freshness was slightly increased by $0,008 \mathrm{mg}$, in pork treated with hydrogen peroxide $(5 \%)$ by $0,295 \mathrm{mg}$, which is 1,04 times higher $(\mathrm{p} \leq 0,001)$, and in pork treated with alkaline detergentdisinfectant $0,645 \mathrm{mg}$, which is 1,08 times more $(\mathrm{p} \leq 0,001)$ compared to control values; and the content of substitutable amino acids in pork of dubious freshness and pork treated with hydrogen peroxide solution and alkaline detergents was significantly reduced respectively by 1,08 times, 1,04 and 1,08 times $(\mathrm{p} \leq 0,001)$.

The total content of essential amino acids in mutton of doubtful freshness was significantly increased by $0,662 \mathrm{mg}$, which is 1,09 times more $(\mathrm{p} \leq 0,001)$, in lamb treated with potassium permanganate solution $(5 \%)$, by $0,292 \mathrm{mg}$, which is 1,04 times more $(\mathrm{p} \leq 0,001)$ compared to control indicators; and the content of substitutable amino acids was significantly reduced by $0,769 \mathrm{mg}$ and $2,078 \mathrm{mg}$ in accordance with the quality of lamb, which is 1,06 and 1,19 times less $(p \leq 0,001)$ compared to the control indicators. The total content of essential amino acids in goat of doubtful freshness was significantly reduced by $0,177 \mathrm{mg}$, which is 1,03 times less $(\mathrm{p} \leq 0,001)$, in goat treated with acetic acid solution $(10 \%)$ by $0,050 \mathrm{mg}$, which is 1,01 times less $(p \leq 0,001)$ compared to control indicators; and the content of substitutable amino acids in the goat of questionable freshness was slightly increased significantly by $0,075 \mathrm{mg}$, which is 1,01 times higher $(\mathrm{p} \leq 0,001)$, and in goat treated with acetic acid was reduced by $0,292 \mathrm{mg}$, which is 1,02 times less $(p \leq 0,001)$ compared to controls. 


\section{REFERENCES}

1. On State Control of Compliance with Food, Feed, Animal ByProducts, Animal Health and Welfare : The Law of Ukraine № 2042-VII, 18.05.2017. $43 \mathrm{p}$.

2. Bogatko N. Safety and quality of slaughtered animal meat when treated with alkaline detergents. Scientific and Practical Journal of Veterinary Medicine, Livestock Technology and Environmental Management. Kharkiv, 2019. № 4. P. 12-18. DOI: 10.31890/vttp.2019.04.02.

3. Hulebak K., Schlosser W. Hazard analysis and critical control point (HACCP) history and conceptual over view. Risk analysis. 2012. № 22 (3). P. 547-552. URL: https://doi.org/10.1111/0272-4332.000383.

4. On basis principles and requirements for safety and quality of food : The Law of Ukraine № 1602-VII from 22.07.2014. Approved. Verhovna Rada of Ukraine. 64 p.

5. The role of hazard- and risk-based approaches in ensuring food safety / S. Barlow et al. Trends in Food Science \& Technology. 2015. Vol. 46. P. 176-188.

6. Birta G. Commodity characteristics of pig production. Nutritional value of pork meat. 2019. URL: https://westudents.com.ua>glavy>90505-8harchovka-tsnnst-mysa-svinins.html.

7. Nutritional and biological value of meat and meat products. 2019. URL: studopedia.org/13-118914.html.

8. Borsolyuk L., Wojciechowska S., Shelkova T. Investigation of amino acid composition and relative biological value of functional meat pates. Innovative development of the food industry : materials VII Intern. Research Practice Conf. November 21, YAN NAR. 2019. P. 19-22.

9. Desarrollo de productos carnicos funcionales: utilizacion de harina de quinua / M. Pena et al. Alimentos, Ciencia e Investigacion. 2015. № 23 (1). P. 21-36.

10. Council Directive EU 89/320/EEC of 9 June 1988 on the approximation of the laws of the EU Member States relating to the inspection and verification of compliance with established rules for laboratories (UPL) (88/320/EEC) to carry out organizational procedures and conditions under which planned, conducted, recorded and presented the results of tests of chemicals used in industry to assess the effects of such substances on humans, animals and the environment. $16 \mathrm{p}$.

11. Council Directive EU № 23/96 of 29 April 1996 on measures to control individual substances and their residual content in live animals and animal products, adopted in violation of EEC Directives 85/358 and Regulation 89/187/EEC and 91/664/EEC. 23 p.

12. Meat. Methods of chemical and microscopic analysis of freshness. GOST 23392-2016. Date of introduction:01.01.1980. Change 10.18.2016. Moscow : Standartinform, 2016. 7 p. 
13. Rules of pre-slaughter veterinary inspection of animals and veterinary-sanitary examination of meat and meat products : Order of the State Department of Veterinary Medicine of 21.06.2002. № 28.46 p.

14. Bogatko N. Control of the safety of meat of slaughtered animals by falsification by express methods : scientific and methodological recommendations.. Bila Tserkva, 2019. 26 p.

15. A method of determining the falsification of meat of slaughtered animals and poultry by formalin treatment / N. Bogatko et al. Patent of Ukraine for utility model 81943, IPC G01N 33/12 (2013.01). № u 2013 02270 ; claimed 02.25.2013 ; publ. 10.07.2013. Bul. № 13. 4 p.

16. The method of determining the adulteration of meat of slaughtered animals and poultry by chlorine treatment / N. Bogatko et al. Patent of Ukraine for utility model 81944, IPC G01N 33/12 (2013.01). № u 2013 02271 ; claimed 02.25.2013 ; publ. 10.07.2013. Bul. № 13. 4 p.

17. The method of determining the adulteration of meat of slaughtered animals and poultry by treatment with hydrogen peroxide / N. Bogatko et al. Patent of Ukraine for utility model 81945, IPC G01N 33/12 (2013.01). № u 201302273 ; claimed 02.25.2013 ; publ. 10.07.2013. Bul. № 13. 3 p.

18. The method of determining the adulteration of meat of slaughtered animals and poultry by treatment with acetic acid / N. Bogatko et al. Patent of Ukraine for utility model 102019, IPC G01N 33/12 (2006.01). № u 2015 03749 ; claimed 21.04.2015 ; publ. 12.10.2015. Bul. № 19. 4 p.

19. The method of determining the adulteration of the meat of slaughtered animals and poultry by treatment with a solution of potassium permanganate / N. Bogatko et al. Patent of Ukraine for utility model 102020, IPC G01N 33/12 (2006.01). № u 201503750 ; claimed 21.04.2015 ; publ. 12.10.2015. Bul. № $19.3 \mathrm{p}$.

20. The method of determining the adulteration of the meat of slaughtered animals and poultry by treatment with alkaline detergents / N. Bogatko et al. Patent of Ukraine for utility model 116831, IPC G01N 33/12 (2006.01). № u 201612243 ; claimed 02.12.2016; publ. 12.06.2017. Bul. № 11.4 p.

21. Tsehmistrenko S., Tsehmistrenko O. The Biochemistry of Meat and Meat Products. Bila Tserkva, 2014. 192 p.

22. Dudchik N. A new method for determining the relative biological value of a protein component of food. Nutrition Problems. 2006. № 4. P. 4-6.

\section{Information about author:} Bogatko N. M.,

Candidate of Veterinary Sciences, Assotiate Professor at the Department of Veterinary and Sanitary Examination

Bila Tserkva National Agrarian University 8/1, Cathedral Square, Bila Tserkva, 09111, Ukraine 